\title{
Ghost Analysis Visualization Techniques for Complex Systems: Examples from the NIF Final Optics Assembly
}

\author{
J. L. Hendrix \\ J. Schweyen \\ J. Rowe \\ G. Beer
}

This paper was prepared for submittal to the Third Annual International Conference on Solid State Lasers for Application (SSLA) to Inertial Confinement Fusion (ICF) Monterey, California June 7-12, 1998

June 26, 1998

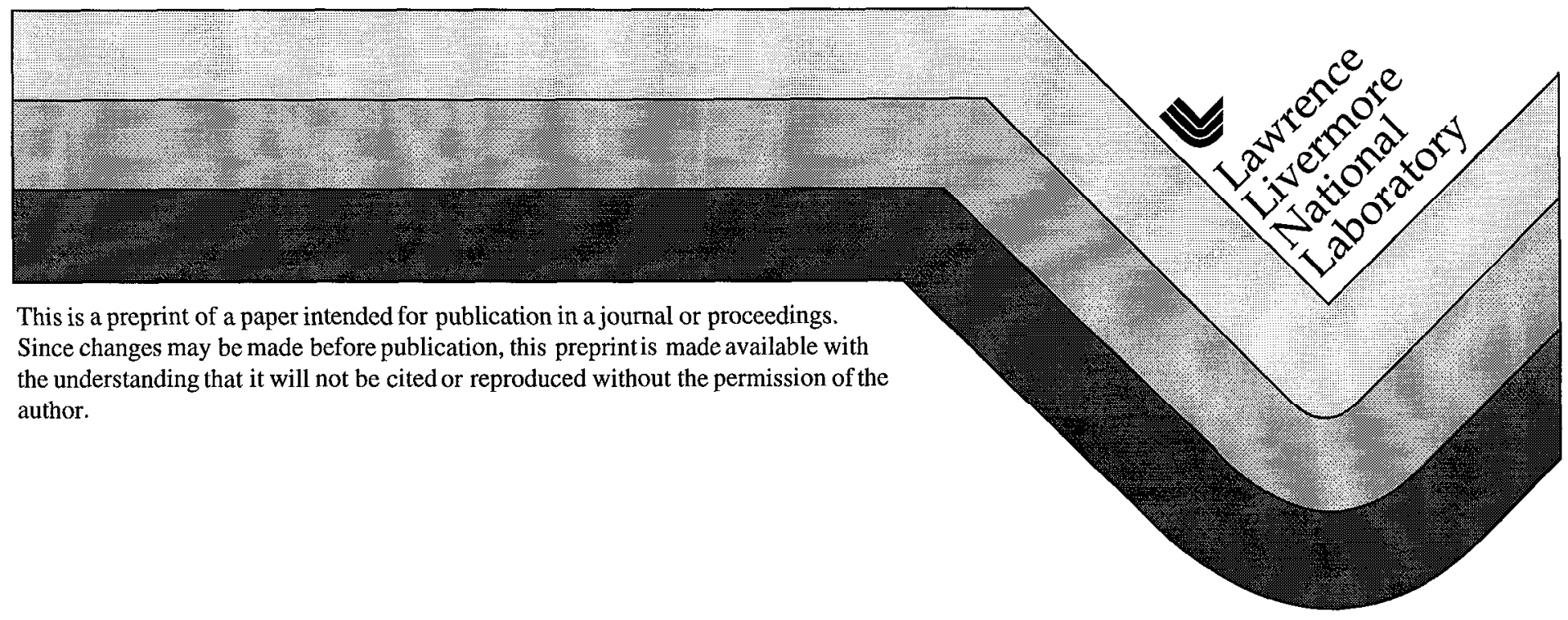




\section{DISCLAIMER}

This document was prepared as an account of work sponsored by an agency of the United States Government. Neither the United States Government nor the University of California nor any of their employees, makes any warranty, express or implied, or assumes any legal liability or responsibility for the accuracy, completeness, or usefulness of any information, apparatus, product, or process disclosed, or represents that its use would not infringe privately owned rights. Reference herein to any specific commercial product, process, or service by trade name, trademark, manufacturer, or otherwise, does not necessarily constitute or imply its endorsement, recommendation, or favoring by the United States Government or the University of California. The views and opinions of authors expressed herein do not necessarily state or reflect those of the United States Government or the University of California, and shall not be used for advertising or product endorsement purposes. 


\title{
Ghost Analysis Visualization Techniques for Complex Systems: Examples from the NIF Final Optics Assembly
}

\author{
James L. Hendrix - AlliedSignal Inc. Federal Manufacturing \& Technologies $\ddagger$, \\ John Schweyen - Breault Research Organization, \\ Jeffrey Rowe - Lawrence Livermore National Laboratory*, \\ Glenn Beer - Lawrence Livermore National Laboratory* \\ Lawrence Livermore National Laboratory \\ POB 808 - L527, Livermore, California 94551
}

\begin{abstract}
The stray light or "ghost" analysis of the National Ignition Facility's (NIF) Final Optics Assembly (FOA) has proved to be one of the most complex ghost analyses ever attempted. The NIF FOA consists of a bundle of four beam lines that: 1) provides the vacuum seal to the target chamber, 2) converts $1 \omega$ to $3 \omega$ light, 3) focuses the light on the target, 4) separates a fraction of the $3 \omega$ beam for energy diagnostics, 5) separates the three wavelengths to diffract unwanted $1 \omega \& 2 \omega$ light away from the target, 6) provides spatial beam smoothing, and 7) provides a debris harrier hetween the target chamber and the switchyard mirrors.

The three wavelengths of light and seven optical elements with three diffractive optic surfaces generate three million ghosts through $4^{\text {th }}$ order. Approximately 24,000 of these ghosts have peak fluence exceeding 1 $\mathrm{J} / \mathrm{cm}^{2}$. The shear number of ghost paths requires a visualization method that allows overlapping ghosts on optics and mechanical components to be summed and then mapped to the optical and mechanical component surfaces in $3 \mathrm{D}$ space.

This paper addresses the following aspects of the NIF Final Optics Ghost analysis: 1) materials issues for stray light mitigation, 2) limitations of current softwarc tools (cspccially in modeling diffractive optics), 3) computer resource limitations affecting automated coherent raytracing, 4) folding the stray light analysis into the opto-mechanical design process, 5) analysis and visualization tools from simple hand calculations to specialized stray light analysis computer codes, and 6) attempts at visualizing these ghosts using a CAD model and another using a high end data visualization software approach.
\end{abstract}

Keywords: ghost analysis, stray light, National Ignition Facility (NIF), final optics,

\section{GHOST ANALYSIS BACKGROUND}

\subsection{Sources of ghosted stray light}

Stray light is unwanted radiation that generally takes the form of scattered light, thermal radiation, or specular reflections (ghost reflections) from nominally transmissive surfaces. Stray light that reaches the focal plane of an imaging system degrades system performance by decreasing signal-to-noise or producing false images. In a high power laser such as NIF, stray light behavior can be critical to system operation. As ghost beams propagate along the beamline they generate additional ghost reflections, and they will also pass through focus. Near focus, the rclatively small amount of energy in the ghost beam becomes concentrated, resulting in potentially damaging fluence levels. Catastrophic failure of optics and metal structures from ghosted stray light has occurred on other laser systems, and can cause runaway damage that would lead to poor system performance and/or poor reliability and availability.

The following definitions should be helpful: 
- $\mathrm{N}^{\text {th }}$ order ghost: a ghost generated by "N" reflections, e.g. a first order ghost is generated by only one reflection, a fourth order ghost was generated by four successive reflections at optical interfaces.

- Ghost family: the ghosts generated by a given sub-set of surfaces. For example, a third order ghost involves the same two surfaces and the additional surface for each individual ghost path is different, thus they are all related but slightly different.

Ghosted stray light includes Fresnel reflections from refractive surfaces as well as unwanted grating orders. In the case of NIF, with 18,000 joules incident on each FOA beam line, even a $0.1 \%$ reflection can be devastating. The focusing optics in the optical train produce $4^{\text {th }}$ order ghosts on the order of $2 \mathrm{~J} / \mathrm{cm}^{2}$, assuming $1 \%$ reflection losses. ( $4^{\text {th }}$ order debris shield ghosts). Diffuse surfaces will randomize the wavefront of a ghost beam and typically render it harmless to the surrounding materials and components. However, this is a generalization and each system and path should be examined in detail.

\subsection{Damage effects table}

The following table gives typical levels of concern when doing ghost analysis on a laser system.

\begin{tabular}{||l|l|}
\hline Energy & Risk / Issue / Concern \\
\hline \hline$>50 \mathrm{~mJ} / \mathrm{cm}^{2}$ & $\begin{array}{l}\text { Liberates adsorbed particles (contaminants) from most surfaces that then can then re- } \\
\text { deposit on optical surfaces, resulting in potential damage sites that can propagate and } \\
\text { cause runaway signal degradation with successive shots. }\end{array}$ \\
\hline$>0.1 \mathrm{~J} / \mathrm{cm}^{2}$ & $\begin{array}{l}\text { Structural metals are ablated/vaporized. The vaporized material deposits on the optical } \\
\text { surfaces generating damage sites that will propagate and cause runaway signal } \\
\text { degradation with successive shots. }\end{array}$ \\
\hline$>20 \mathrm{~J} / \mathrm{cm}^{2}$ & $\begin{array}{l}\text { Optical coatings fail, degrading the magnitude and quality of the transmitted optical } \\
\text { signal } \\
\text { At this fluence optical glass materials will begin to vaporize, metals and polymers will } \\
\text { explosively ablate }\end{array}$ \\
\hline$>1000 \mathrm{~J} / \mathrm{cm}^{2}$ & $\begin{array}{l}\text { Causes air breakdown (strips electrons off of gas molecules creating a plasma) under } \\
\text { many conditions. Energy is absorbed or scattered by the plasma causing loss of signal } \\
\text { and degradation of signal quality. } \\
\text { Optical materials will ablate. Most metals and polymers will explosively ablate \{for } \\
\text { very clean dry air, 1 nS pulse @ 1 micron\} }\end{array}$ \\
\hline
\end{tabular}

The liberation of trapped contaminants will be an issue on the NIF FOA because there are large areas on mechanical surfaces exposed to fluences in excess of $50 \mathrm{~mJ} / \mathrm{cm}^{2}$. The issue of ablating structural materials is a serious one in that it will lead to collateral damage on the optics. While the precise value of this varies with the specific material this is the level at which material specifics must be carefully considered. The air breakdown value can be significantly less than the $1000 \mathrm{~J} / \mathrm{cm}^{2}$ that is listed because it is highly dependent on the pressure, humidity, and level of contaminants in the air/gas under test. In tests with optical fibers the author has experienced air breakdown as low as $80 \mathrm{~J} / \mathrm{cm}^{2}$.

\section{FINAL OPTICS ASSEMBLY OPTICAL DESIGN}

The NIF Final Optics Assembly (FOA) is broken up into four beam lines. The optics for each beam line and it is associated mounting hardware and enclosure is referred to as an Integrated Optics Module (IOM). Figure 1 shows the final optics for a beam line. 


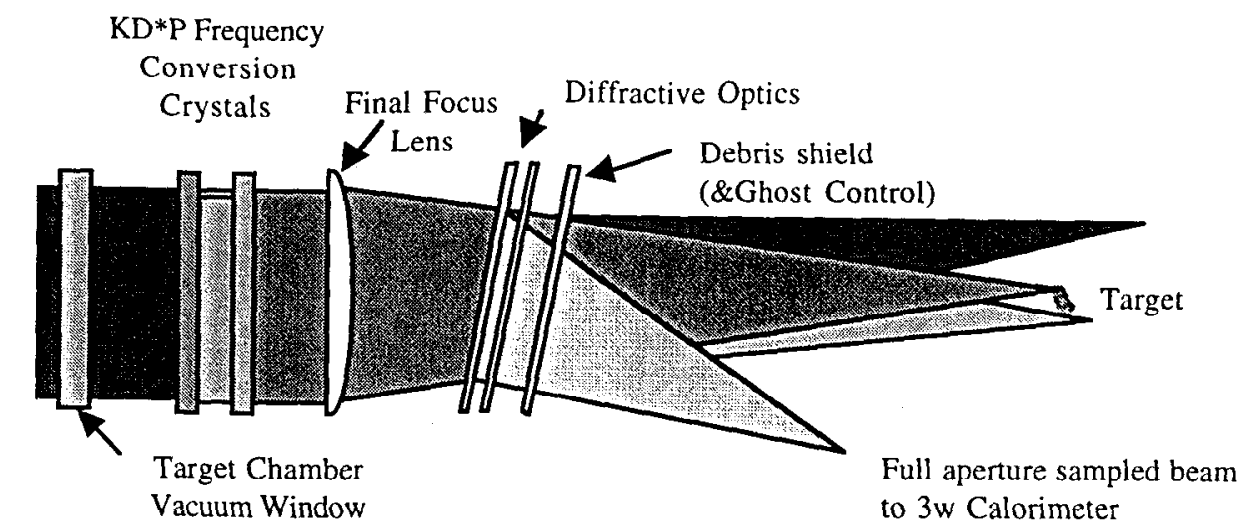

Figure 1: The optical train for a single beam line of the NIF Final Optics Assembly shows the components and optical functions for each.

The final optics assembly performs several functions on the NIF. The Target Chamber Vacuum Window (TCVW) provides both vacuum isolation of the target chamber and ghost control via its tilt angle (not shown in this view (see Figure 3\}). The frequency conversion crystals use a type I/Type II sum frequency generation scheme using $\mathrm{KDP} / \mathrm{KD} * \mathrm{P}$. The final focus lens provides for the energy to be focused at the target chamber center. The FFL focal length is $7703 \mathrm{~mm}$ and the lens is inserted in the reverse orientation to what is normally done in optics with the flat side pointed toward the incoming beam. This is done to provide for a $3 \mathrm{w}$ diagnostic reflection that verifies proper orientation of the frequency conversion crystals rclative to the incoming beam. Following the frequency conversion crystals there is significant residual $1 \omega$ and $2 \omega$ light (not shone explicitly until after the diffractive optics). The diffractive optics perform three function in the NIF, each function being provided by separate diffractive structure. These gratings and their generic functions are shown in Figure 2. The Beam Sampling Grating (BSG) is a holographically produced diffractive structure that provides a calibrated sample of the $3 \mathrm{w}$ beam to be diverted to a calorimeter for energy measurement. The Color Separation Grating (CSG) diverts the unconverted $1 w \& 2 w$ light from the target. Note only $t$ first diffracted order is shown in Figure 1 for both the BSG and the CSG. The other orders represent ghost energy that must be mitigated.

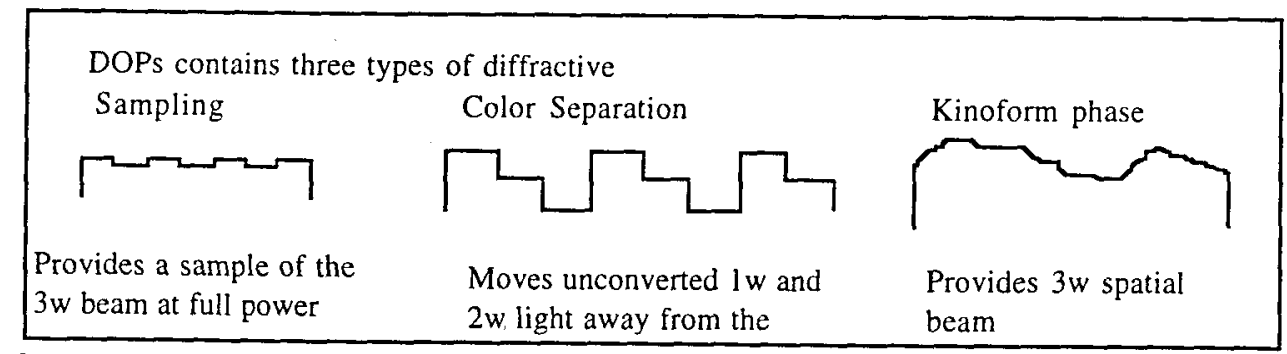

Figure 2: The diffractive optic structures are shown for the beam sampling grating, color separation grating and kinoform phase plate.

Figure 3 shows the FOA and its IOMs with optical components identified as well as acronyms defined. 


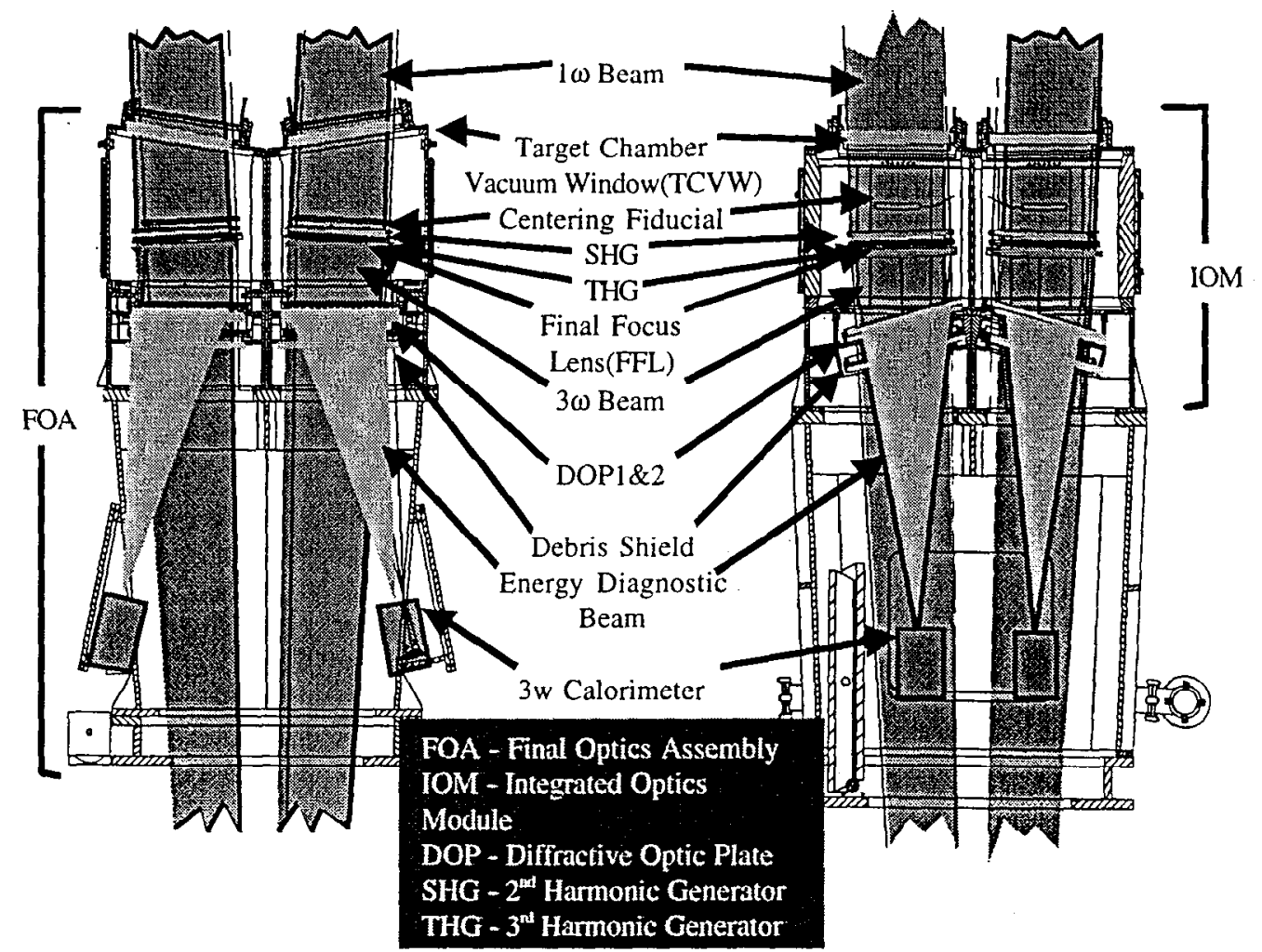

Figure 3: The opto-mechanical design of the NIF Final Optics Assembly shows the components and optical functions for each.

The added complexity of the mechanical structure for any system is a challenge, especially when combined with the lower damage threshold of most structural materials.

\section{GHOST CHALLENGE IN THE NIF FOA}

The number of ghosts generated by a series of refractive elements is asymptotically approximated by (M_SURF) ${ }^{\mathrm{N}-S P L I T}$ where M_SURF is the number of transmissive interfaces, and N_SPLIT is the number of ghost orders considered. Each IOM contains seven refractive optical elements, comprising 14 surfaces. Two of the three diffractive surfaces generate unwanted grating orders, each of which is equivalent to an additional splitting surface. To complicate matters, off-axis ghosts from tilted elements are generally broken into pieces as they encounter lens edges. As mentioned above, ghosts up through fourth order present damage risks to the FOA opto-mechanical structure. The $3 \omega$ beam in the FOA will therefore generate on the order of 500,000 ghosts whose fluence and location must be quantified.

Unconverted $1 \omega$ and $2 \omega$ light carries approximately $40 \%$ of the total energy. Additionally, $15 \%$ of the $3 \omega$ energy may be reflected back towards the IOM via Stimulated Raman Scattering (SRS) and Stimulated Brillouin Scattering (SBS). Because of chromatic dispersion at the diffractive structures, these various beams generate ghosts that focus at different locations. The bottom line is that the FOA ghost analysis entails the computation of several million ghosts.

Ghosts are filtered at several stages based on degree of collimation, fluence, and focus location. The optics and mechanical structures intercept approximately 50,000 ghosts that exceed the analytical fluence threshold at the surface or at geometric focus. These are tabulated in an Excel spreadsheet. Only a very small fraction of these focus close enough to a surface to contribute significantly to surface fluence. 
The large numbers of ghost paths and large energy transiting the system in the NIF FOA combined with the relatively large size of the assembly yield some demanding requirements for ghost analysis. The Integrated Optics Module (IOM), which represents one beamline, is approximately $0.5 \mathrm{M} 3$. Although the point spread function for badly aberrated ghosts have rms diameters on the order of $1 \mathrm{~mm}$; peak fluence is usually confined to regions on the order of 10 microns in diameter. Since the ghost at focus can be smaller than 10 microns it would require 1013 computational cells to address even a 10 micron resolution in a regularly distributed array. This would require approximately 250 terabytes of storage. One solution is to represent the volumetric fluence distribution in point cloud form and to display the information using point glyping, as described below.

\section{GHOST ANALYSIS \& VISUALIZATION TECHNIQUES}

Several methods were used in the ghost analysis project for NIF, some of which will be described in the following sections. Those that are not specifically listed include CODE V, GLAD, and IDL.

\subsection{First order optics and the English method}

The best starting point for any such analysis is first order optics. By examining the optical system and identifying the bounding ghosts much can be done to identify and mitigate problem ghost paths. We uses a simple spreadsheet approach to ray tracing that allowed us to determine the focus positions of the defining ghost paths over the extremes of travel of the final optics cell (FOC). The FOC contains the frequency conversion crystals and the final focus lens in the NIF final optics. The English method is a means of graphically displaying this type of data. It is named for its creator R. Ed English. The method is shown in Figure 4 and illustrates how the ghost path focus position is displayed for FOC travel position. Note that the Bars represent the range of travel of the ghost with FOC position. 'I he dot in the bar represents the ghost focus position at the nominal focus position for the FOC. The " + " represents the position as the FOC is moved in the forward direction and conversely the "." shows the position as the FOC moves in the reverse position.

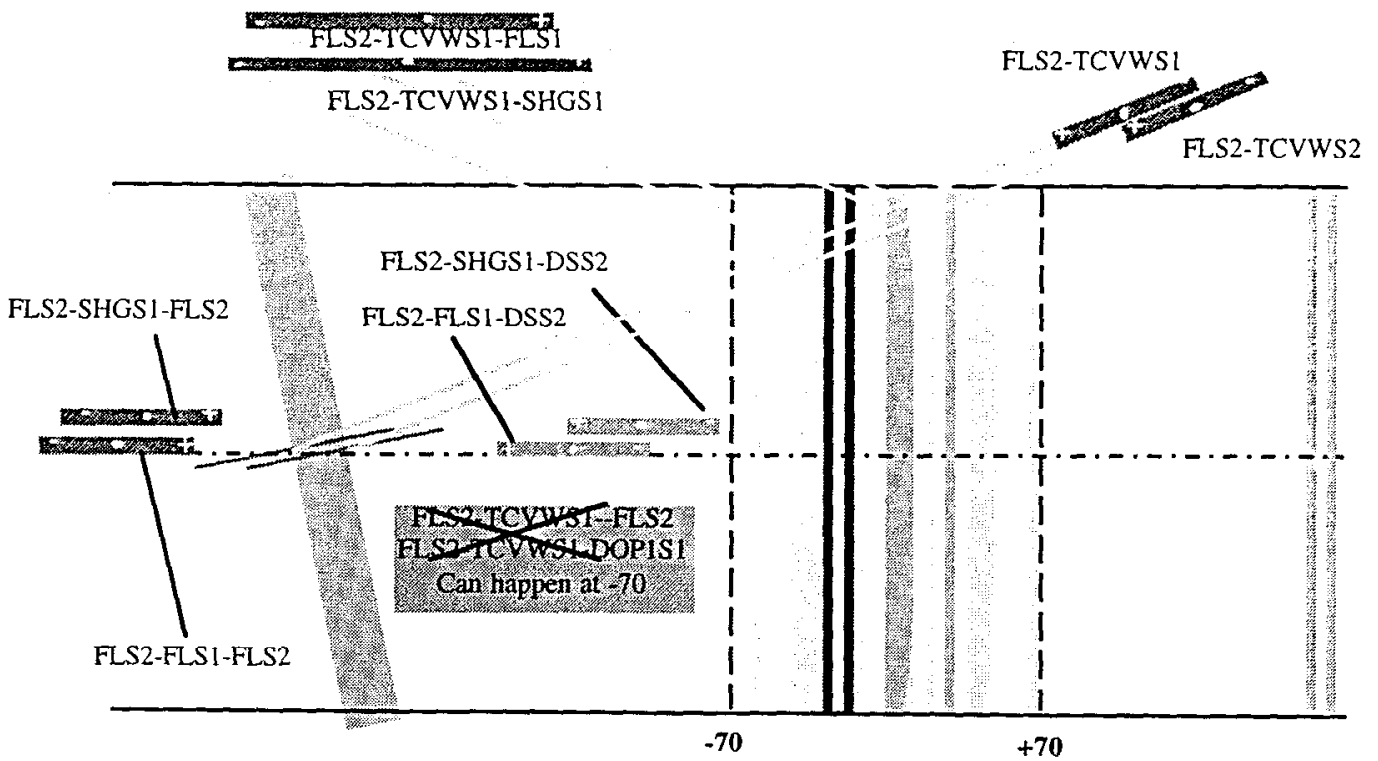

Figure 4: The English method of ghost path visualization allows for ghost focus locations to be shown as a function of optical system zoom positions. ASAP

The FOC position is shown in light gray for the two extreme focus positions. This analysis was performed when one Diffractive Optic Plate (DOP) was in the FOC; this has been moved to a DOP cassette in the current design. The path naming convention is the element acronym such as TCVW is the target 
vacuum window, followed by the side number one being toward the input side relative to the laser. Thus FLS2-SHGS1-DSS2 is the second surface of the focus lens (the curved surface) followed by a bounce off of the second harmonic generator and then the debris shield second side (toward the target). See Figure 3 for acronyms definitions.

\subsection{AS AP}

ASAP (Advanced Systems Analysis Program) is a general optical analysis software tool that can be used to evaluate stray light performance. The program kernal is a non-sequential ray tracer that automatically tracks and quantifies all possible ghost paths up to some user-specified level. Optical and mechanical components are integrated into a single model. The program reports ghost fluence at designated surfaces and optionally records statistics on ghost foci between surfaces.

For the FOA analysis, ghosts are initially evaluated using an algorithm based on geometric optics that was specifically developed to overestimate peak fluence. A ghost beam is flagged as potentially threatening if its fluence at a surface or at focus exceeds user-specified thresholds. Peak fluences of energetic ghosts are then more accurately computed using the program's wave optics capability. Since peak fluence and location thereof depends on beam aberrations, which are typically on the order of hundreds of waves, it is necessary to search through a small volume in the vicinity of geometric best focus. The FOA grating surfaces and tilted optics preclude the use of symmetry in this calculation.

ASAP's graphics capability is not well suited to the display of sparsely distributed data over large volumes. The display of accumulated ghost fluence throughout the FOA optical train is therefore handled off-line. Off-line processing also allows first-order tolerancing to be considered as described below.

\subsection{ASAP \& PRO-E}

One attempt to visualize the ASAP results was to import the ghost focus locations (in $x, y, z$ ), direction cosines indicating the direction the ghost was traveling at focus, and the distance from the last optical surface into a mechanical design program. PRO-E was used for the mechanical models and thus the ASAP data was imported into PRO-E as a table generated pattern. The results of that visualization are shown in Figure 5.

This visualization technique has tremendous potential however the lack of access to the color table in PRO-E (in order to show fluence data) and the need for significant operator input to perform sectioning operations at surfaces of interest are currently severe limitations to its use.

Of course the ability to use the actual mechanical geometry and have it update as changes are made during the design is a definite advantage. This coupled with the ability to rotate, section and zoom provides for a powerful analysis tool. Unfortunately time and manpower constraints did not allow for the limitations to be addressed and

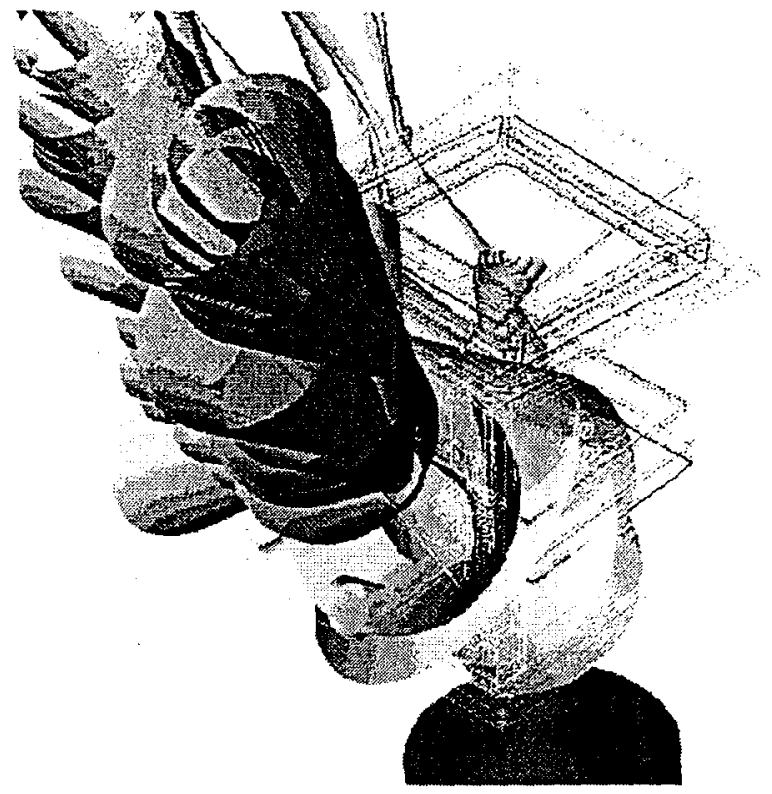

Figure 5: Importing the ASAP calculated ghost data results into PRO-E provides a good visualization of the ghost interactions with the mechanical features of an IOM. However the lack of fluence data at the mechanical surface s limited the usefulness of this approach. 
overcome.

\subsection{Post processing}

It became apparent early in the project that a method to post process the data that would allow for the integration of the various colors was necessary. It was also envisioned that a tool that would provide output similar to a finite element analysis model would be ideal for visualizing the FOA ghosts.

The ASAP data was post processed using a FORTRAN 90 program that performs the following:

$$
E_{(x, y, z)}=\left\{\underset{d f}{\operatorname{MAX}} \sum_{\lambda_{i}} \sum_{n=1}^{n g h o s t s} \underset{S U R F_{k}}{\operatorname{SNAP}}\left[E n\left(x, y, z ; \lambda_{i}, d f, S U R F_{k}\right)\right]\right\} \cap \text { Geometry }
$$

Where:

$$
\begin{gathered}
E_{n}\left(x, y, z: \lambda_{i}, d f, S U R F_{k}\right)=\left\{\begin{array}{lc}
\text { Estimated Peak Surface Fluence } & \left|\vec{P}_{\text {focus }}-\vec{P}_{\text {center }}\right|<d_{\text {tolarance }} \\
\text { GeometricFocus } & \left|\vec{P}_{\text {focus }}-\vec{P}_{\text {center }}\right| \leq d_{\text {tolarance }}
\end{array}\right\}, \\
\text { Surface Fluence }
\end{gathered}
$$

Estimated peak fluence at geometric focus $=200\left(\frac{\varphi_{n}}{\sigma^{2}}\right)$.

Surfacefluence $=\frac{\varphi_{n}}{A_{\text {footprint }}}, \varphi_{n}=$ Energy $n^{\text {th }}$ ghost, $\sigma=$ RMS blur diameter @ geometric focus. $A_{\text {foutpr int }}=$ Area of ghost footprint @ surface.

And $d f$ is change in focus position.

Thus the ghost locations and fluences are calculated using ASAP. The fluence from overlapping ghost and wavelengths are integrated. The ghosts that have their focus position within a given distance from a surface are snapped to that surface ( $15 \mathrm{~mm}$ is a typical snapping distance). And finally, the maximum for each cell from the different focus positions is taken for each computational element. 
The result of the post processor is then ready for import into a visualization tool such as IBM's Data Explorer or IDL.

\subsection{Visualization with IBM Data Explorer (IBM DX)}

A visualization software package like IBM DX allows data from the post processed ASAP data to be imported and overlaid with a geometry file. The data points from ASAP are also glyphed. Glyphing allows the data resolution to be filled in by making the point a cube with, in our case, a one centimeter face dimension. The resultant visualization is shown in Figure 6.

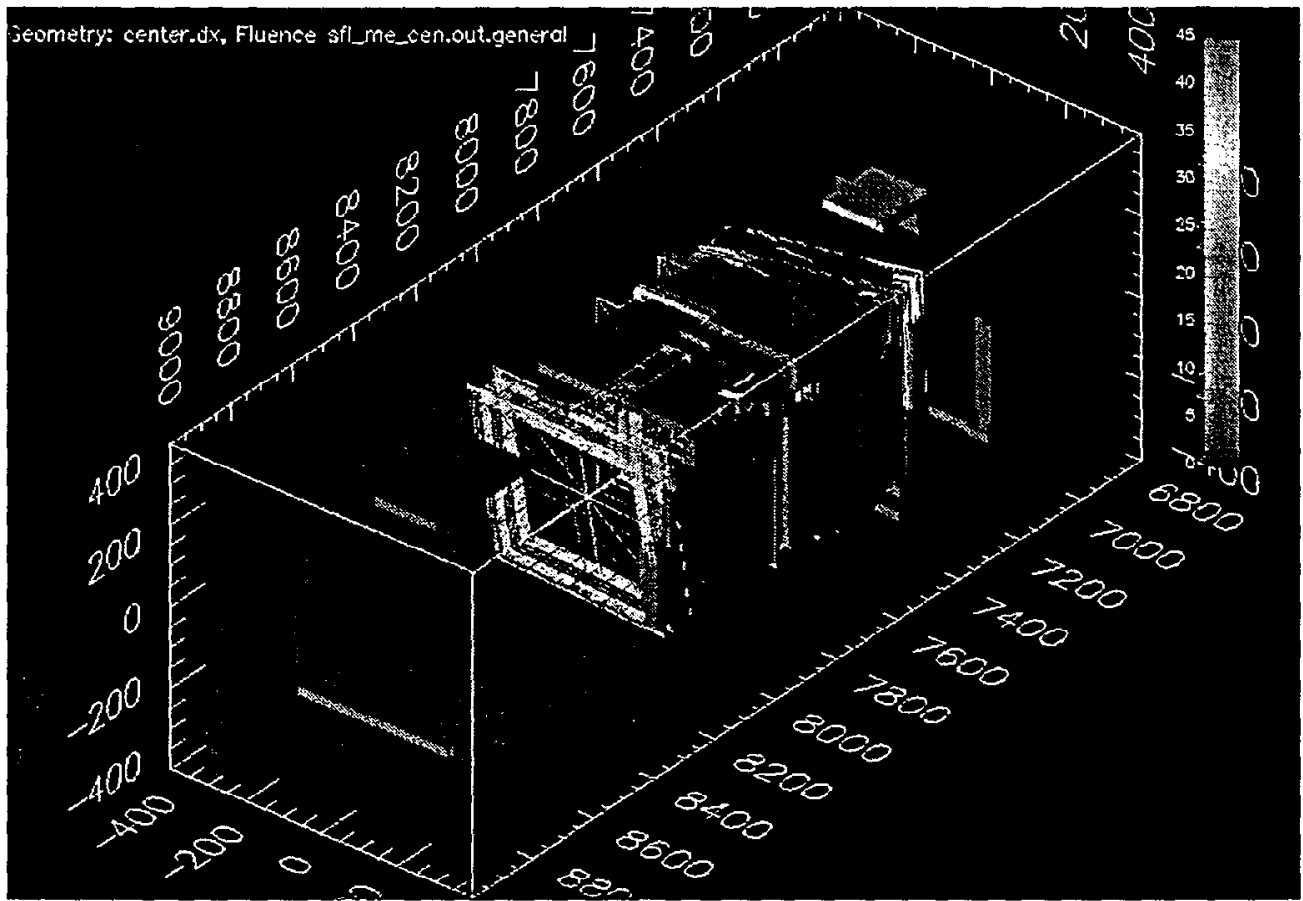

Figure 6: The visualization tool allows geometry and ghost fluence data to be shown simultaneously and clearly indicating where the areas of concern are located.

Other features of a visualization tool are the ability to clip ghosts outside of user selected limits. Figure 7 shows a clipped view with only ghosts above $4 \mathrm{~J} / \mathrm{cm}^{2}$ shown.

Figure 8 shows only ghosts between 0.5 and $4 \mathrm{~J} / \mathrm{cm}^{2}$. In addition it shows the path information which must be retrieved using spreadsheets based on the fluence and location. The paths shown are for the dominant ghost paths for each ghost patch. A ghost patch is a superposition of overlapping ghosts footprints (as well as wavelengths and focus positions).

Geometrically calculated ghost fluences tend to overestimate the actual fluence and thus coherent evaluations for hot ghost

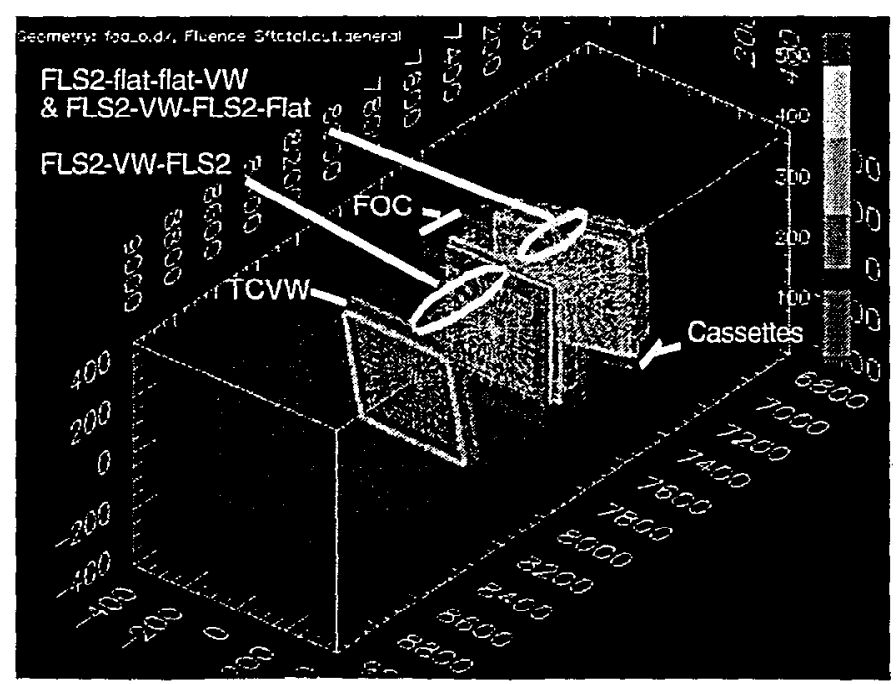

Figure 7: The NIF IOM is rendered with only ghosts above $4 \mathrm{~J} / \mathrm{cm}^{2}$ shown. This can be defined for any fluence range of interest. 
paths are calculated using ASAP or GLAD. Figure 9 Shows several ghost patches in the most recent analysis runs with the dominant paths indicated, the geometrically calculated fluence and the coherently calculated fluences. The geometrically determined fluence is almost always lower than the coherently determined fluence. It is important not to assume that it is always true however, we have found a few paths where the geometrically determined fluence was lower. The difference ranges typically from a factor of 3 to a factor of an hundred.

The geometry can be selectively excluded to zoom in on a particular area, but this is done prior to importing into the visualization program and must be accompanied by a range limited (spatial) set of ghost data. Figure 10 shows an example of the ghost data and the optical elements only and is very useful for identifying stay out zones. Note that the data shown if Figure 10 includes fluences on optics and the focus positions of the ghost paths with

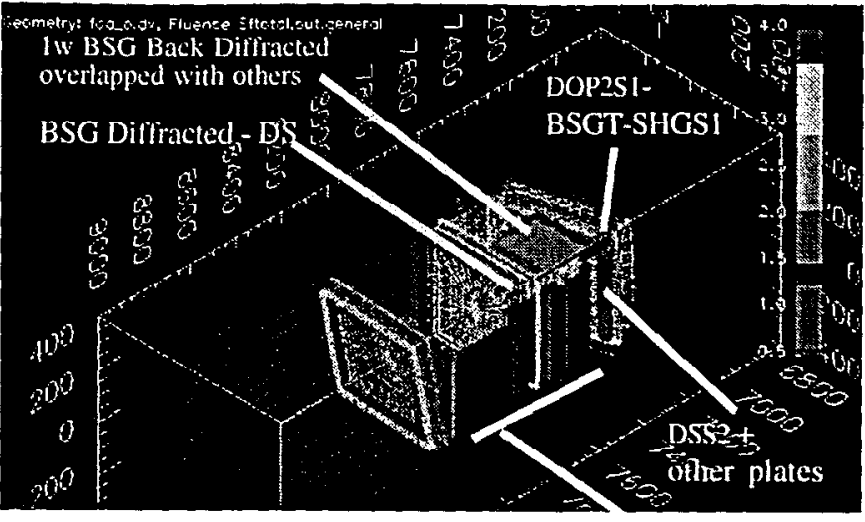

Figure 8: Ghosts within the range of 0.5 to $4 \mathrm{~J} / \mathrm{cm}^{2}$.are shown. Also note the path information for the major ghost patches which indicate the dominant contributor for each patch.

fluences greater than $1 \mathrm{~J} / \mathrm{cm}^{2}$. The solid areas over the wire frame of the optics are all less than $3 \mathrm{~J} / \mathrm{cm}^{2}$ and tend to obscure other ghost information this can be clipped to show only those ghost above and or below user specified fluences. It is interesting to note the range of fluences in this plot. The $1^{\text {st }}$ order ghost of the curved surface of the lens has a coherently determined fluence of $5 \mathrm{MJ} / \mathrm{cm}^{2}$. All of the plots shown are in the same rotation angle, however the data can be rotated, panned, and zoomed to interrogate various portions of the system.

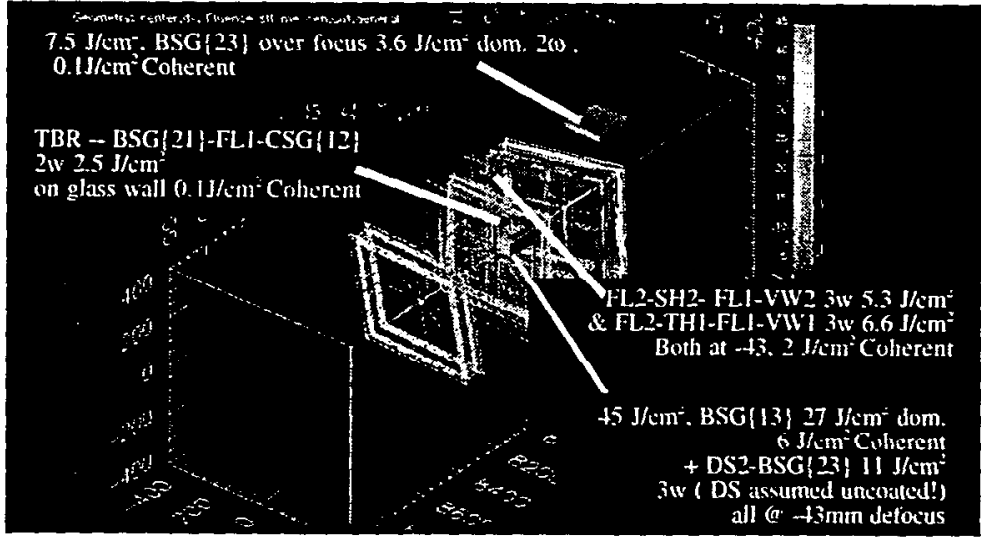

Figure 9: Ghosts from the most recent analysis runs greater than 2.5 $\mathrm{J} / \mathrm{cm}^{2}$ are shown. Note the path information for the major ghost patches, which indicate the dominant contributor for each patch as well as the geometrically and coherently determined fluences for the dominant path

Figure 11 shows the optical elements again, this time with the ghosts less than $3 \mathrm{~J} / \mathrm{cm} 2$ and greater than $100 \mathrm{~J} / \mathrm{cm} 2$ clipped out. Note that the color scale for fluence is more useful than in Figure 10.

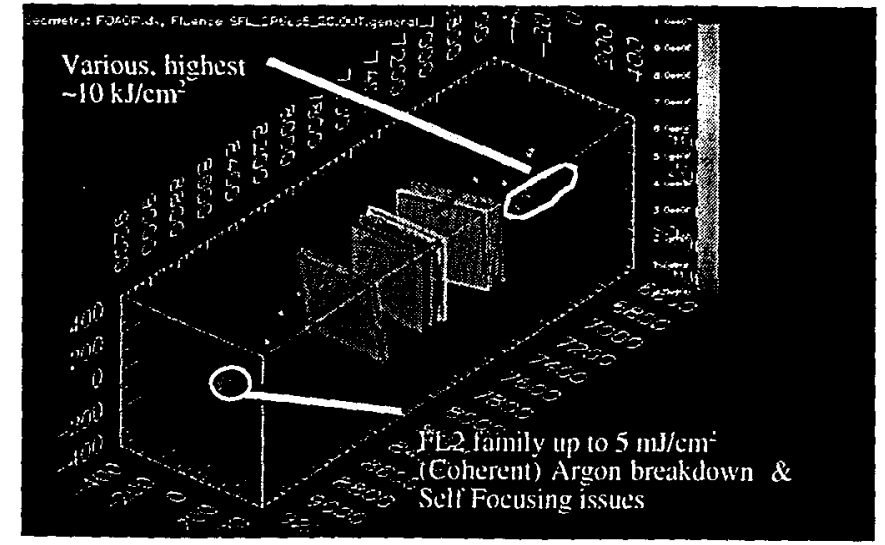

Figure 10: The NIF FOA optics are shown (in wire frame) with the ghosts both at the surface of each optic and the fluences at focus shown. This represents in excess of 24,000 ghosts above $1 \mathrm{~J} / \mathrm{cm}^{2}$ tracked for the optics 


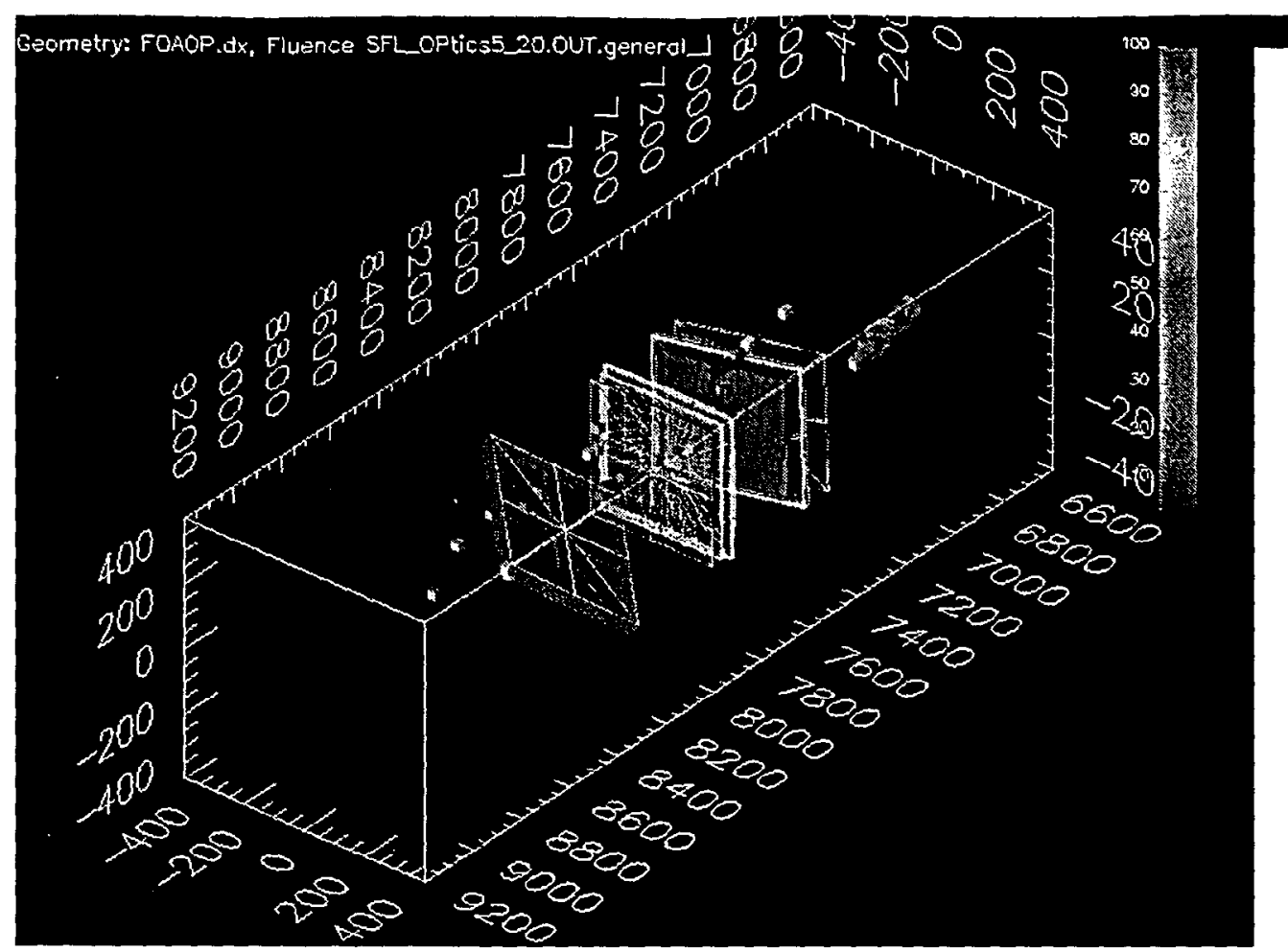

Figure 11: The NIF final optics elements are shown combined with the ghost footprints on the optics and ghost focus positions between 3 and $100 \mathrm{~J} / \mathrm{cm}^{2}$.

\subsection{Tracking back to ghost paths}

The most important data provided by a stray light analysis are: 1) how much stray light reached the critical surfaces, and 2) how did the stray light propagate to the critical surfaces. It is the second item that allows one to decide which design changes are needed to improve performance. In the context of ghost analyses, this information is provided by specifying the sequence of ghost reflections encountered by the beam on its way to the critical surface. This sequence is referred to as a ghost path.

ASAP reports path information for all ghost beams at all critical surfaces. At each element of the displayed volume, the post-processor determines which path contributed the most stray light. The spatial coordinates of high fluence areas observed in IBM DX are associated with a path index, which in turn corresponds to a ghost path sequence in the Excel ghost catalog. The ghost path data is dialed back into ASAP to obtain a graphical raytrace, a coherent estimate of peak fluence, or sensitivity data.

\subsection{Mitigation Techniques}

Ghost mitigation techniques for optical elements and the surrounding mechanical structures differ significantly. To mitigate the ghosts that fall on optical surfaces it is usually sufficient to change the optical element spacings or tilt angles of the elements. This tends to put those ghosts onto the mechanical structure. Since engineers have yet to figure out how to float the optics in space while maintaining the precision alignments required for lasers systems there are always mechanical structures required to hold and position the optics. These mechanical structures tend to be made from materials that have far lower laser damage thresholds (LDT) than those of the optical elements. As a result, either the material for the mechanical hardware must be changed or it must be protected with a material that can withstand the ghost fluence. 
The most widely used technique for ghost mitigation is to cover the structures with absorbing glass. This has two distinct drawbacks. First, the absorbing glasses (e.g. NG4) are expensive. Second these glasses are polished. The polishing is an issue for two reasons. The $3 \omega$ laser damage threshold (LDT) of polished glass surfaces tends to be a strong function of the polishing techniques employed. And in the NIF FOA the ghosts arrive at the majority of optical surfaces at very high angles of incidence. The high angle of incidence results in ghost energy being reflected (dependant on incoming polarization). The NIF FOA analysis complexity was such that, given current operating constraints, the system could not analyze the extra surfaces created when mechanical structures were covered with glass. Further and more importantly in general is that these reflected ghost paths can be redirected to other areas of the system and cause laser damage to occur.

In order to mitigate these ghosts an architectural glass was found with favorable absorption characteristics. Since it is a float glass a "polished" surface is obtained without mechanical polishing yielding a consistent LDT. In addition, a chemical etch was found that allows the material to be a diffuse reflector, and thus break up the ghost spatial profile, and still have acceptably high LDT. The LDT for the chemically etched glass was about a factor of 2 higher than for the mechanically roughened surface.

Other materials such as pure Alumina ceramics and polymers (e.g. Teflon) are being investigated where glass is not desirable from an assembly and reliability perspective.

One other technique that was used is to allow high flucnec ghosts to pass through a hole or slot in an absorbing glass plane and thus allow the ghost to pass through the material and through focus. The energy is then absorbed once the beam is sufficiently past focus so that the fluence is below the LDT of the absorber.

Other methods include limitations on operations. Examples of this on NIF would include limiting the range of travel of the FOC in one direction or the other and limitations on the pointing of the beam away from target chamber center. Both of these methods were used, however they did not violate the NIF requirements.

\section{CONCLUSIONS AND LESSONS LEARNED}

Many analysis tools for ghost identification and visualization have been used in the NIF Final Optics Assembly. While limitations and complications exist that hinder the analysis efforts, this project represents one of the most elaborate ghost analyses ever performed. By choosing parameters appropriately and post processing the ghost data high confidence is obtained in identifying and quantifying all major ghost paths.

There are many visualization tools to choose from and they are essential in highly complex systems such as the NIF FOA. Mitigation tools are somewhat limited but are sufficient for most tasks. The biggest hindrance to mitigation is the uncertainty in laser damage thresholds of structural materials.

One caution to users of diffractive elements is that the higher order, forward and backward, diffracted grating orders must be included in the analysis. We found several hot ghost paths involving such grating orders and have found orders that overlap at different wavelengths.

One additional caution is that the use conditions of the system must be analyzed. An example from the NIF is that the system is required to point off target chamber center by several centimeters. The angular changes to the beam and optics to accommodate that function greatly impacted the mitigation approaches in the FOA.

Motherhood on stray light: Important to evaluate threatening ghosts during the design phase. (It is generally more cost efficient to identify damaging ghosts analytically rather than after the hardware is built.) 
Anti-reflection coating performance drives ghosted stray light performance - must maintain good coating performance.

Analytical tools:

In the context of stray light analysis for pulsed lasers, the ideal software would have the following capabilities:

1) display of volumetric stray light data

2) user-friendly technique for modeling arbitrary holographic patterns over large surfaces

3) fast evaluation of coherent fields (or faster computers)

An integrated analysis tool would be most welcome in this type of work, however the pieces exist to adequately perform the required analysis at present. No doubt, the computer industry will enable this type of analysis to be done more quickly and more efficiently in the future as well.

\section{ACKNOWLEDGMENTS}

All work performed in support of this research was funded by the U. S. Department of Energy and performed by the University of California under Contract No. W-7405-Eng-48.

$\ddagger$ AlliedSignal Federal Manufacturing \& Technologies is operated for the U. S. Department of Energy by AlliedSignal Inc. under Contract No. DE-AC04-76-DP00613.

* Lawrence Livermore National Laboratory is operated for the U. S. Department of Energy by the University of California under Contract No. W-7405-Eng-48.

Due to the poor black and white reproduction quality from the color originals a color electronic version of this paper is available on request in Microsoft Word format by e-mailing jlhendrix@llnl.gov. 